\section{Differential kinetics of plasma procalcitonin levels in cerebral malaria in urban Senegalese patients according to disease outcome}

\author{
Babacar Mbengue, ${ }^{1}$ Bacary Diatta, ${ }^{2}$ \\ Birahim Niang, ${ }^{2}$ Ngor Diagne, ${ }^{1}$ \\ Mamadou Ndiaye, ${ }^{2}$ Laurence Marrama, ${ }^{3}$ \\ Ronald Perraut, ${ }^{1,4}$ Alioune Dieye ${ }^{1}$ \\ ${ }^{1}$ Laboratoire d'Immunologie Faculté de \\ Médecine, Université Cheikh Anta Diop \\ de Dakar; ${ }^{2}$ Hôpital Principal, Service de \\ Réanimation, Dakar; ${ }^{3}$ Unité \\ d'Immunologie, Institut Pasteur de Dakar, \\ Senegal; ${ }^{4}$ Institut Pasteur, Division \\ International, Paris, France
}

\section{Abstract}

$P$. falciparum malaria continues as the serial killer of over a million lives yearly, mainly for children in sub-Saharan Africa. For severe malaria, we are still on the quest for a prognostic marker of fatal outcome. We analysed the association between serum levels of Procalcitonin (PCT), a marker of septic inflammation, and clinical outcome in Senegalese patients admitted with confirmed cerebral malaria in the intensive care facility of Hopital Principal. A total of 98 patients living in the hypoendemic urban area of Dakar, Senegal, were enrolled during transmission seasons. Levels of PCT were compared between surviving vs the $26.5 \%$ fatal cases in blood samples of the 3 days following hospitalisation. Mean PCT levels were elevated in patients with active infection, with a large range of values ( 0.1 to 280 nanog per $\mathrm{mL}$ ), significantly higher on day 0 in fatal cases than in surviving (53.6 vs 27.3; $\mathrm{P}=0.01$ ). No exact individual threshold level could indicate occurrence of fatality, however mortality could be most accurately predicted by PCT level above 69 nanog per ML and there was a very clear different profile of evolution of PCT levels on the 3 days of observation decreasing early from day 1 in surviving patients $\left(\mathrm{P}<10^{-3}\right)$, contrary to fatal cases. These results indicate that PCT kinetic rather than intrinsic level could be of use to predict a reduced risk of fatality in patient with cerebral malaria and could serve as potential predicting marker for severe malaria.

\section{Introduction}

Malaria due to Plasmodium falciparum ( $P$. falciparum), one of the leading infectious killers of tropical and sub-saharan populations of the third world, is a markedly heterogeneous disease ranging from mild manifestations with complete recovery to death. In non-immune individuals, early diagnose and appropriate treatments and follow-up of patients leads to definitive recovering within a few days. Conversely, a late diagnosis and/or an inadequate delayed therapy may lead to the development of clinical complications such as anaemia, cerebral malaria, pulmonary and renal failures. An important challenge for attending physicians in the management of $P$. falciparum malaria is to identify patients who are at high risk of complications and mortality requiring particular intensified care and treatment. Importantly, clinicians in endemic settings have to act urgently with a limited panel of information on the exact sequence of events that happened before occurrence of severe accesses before hospitalization. In such situation, predictive markers of clinical infection are needed to help identify such patients with high risk of fatal outcome. In tropical countries, as antimararial treatments are often taken by individuals during the transmission season, level of parasitaemia on day 0 of admission is of limited value as predictive criterion of outcome. Therefore, other predictive markers are necessary.

Procalcitonin (PCT), a 116 amino acid protein precursor of the hormone calcitonin (CT, 32 amino acids), is considered to be an important clinical marker for sepsis, systemic inflammatory reactions that are triggered by pathogens such as bacteria, fungi and parasites, including P. falciparum. ${ }^{1}$ In general terms, PCT levels correlate with the severity of infection and sepsis, ${ }^{2}$ possibly mediated through the effect of endotoxin-like mechanism. ${ }^{3}$ Recent studies have shown that PCT levels also correlate with parasitaemia and severity of adult malaria. ${ }^{4}$ We focused the investigation on this single marker for practical and applicable cost reasons, as complex biological markers from pro-inflammatory cascade are not in the routine procedure for patients followup. To further analyse the potency of such marker for complicated cerebral malaria, a retrospective recruitment was performed including 98 well-categorized hospitalized individuals, presenting symptoms of confirmed cerebral malaria with or without fatal outcome (followed-up in the intensive care facility) on which levels of PCT have been measured.

\section{Materials and Methods}

\section{Sample collection and study popu- lation}

Hospitalized subjects were living in the hypoendemic urban area of Dakar, Senegal.
Correspondence: Dr Ronald Perraut, Division International, Institut Pasteur, 28 rue du $\mathrm{Dr}$ Roux, 75015 Paris, France.

Tel. +33.01.45.68.82.11.

E-mail: ronald.perraut@pasteur.fr

Key words: cerebral malaria, Procalcitonin, fatality risk, endemic region.

Acknowledgements: the authors wish to acknowledge all the medical staff from Hopital Principal for its participation to this work. We thank also Drs T Ka and R Kane for participation for sample management and patient recruitment. This work was supported by grants from Institut Pasteur Fundation and Ministère de la Recherche et de la Technologie.

Contributions: the study was initiated by RP and $\mathrm{AD}$, with the help of $\mathrm{BN}, \mathrm{BD}$ and $\mathrm{MN}$, field work, collection of serum samples and clinical data was realized by BM and ND; LM performed the statistical analyses.

Conflict of interest: the authors report no conflicts of interest.

Received for publication: 8 July 2011.

Revision received: 11 October 2011.

Accepted for publication: 2 November 2011.

This work is licensed under a Creative Commons Attribution NonCommercial 3.0 License (CC BYNC 3.0).

CCopyright B. Mbengue et al., 2011

Licensee PAGEPress, Italy

Microbiology Research 2011; 2:e22

doi:10.4081/mr.2011.e22

They were admitted in the intensive care facility of Hopital Principal where treated for confirmed cerebral malaria. More than 2 millions people live in the urban area of Dakar, they are exposed to low and variable increasing infection risk: less than 0.5 and, recently in some places, around 3 to 9 infective bite per individual per year during the rainy season, transmission is characterized by a highly variable density of vector. ${ }^{5,6}$ A mean incidence of $2.4 \%$ of clinical accesses has been observed. ${ }^{7}$ The samples have been previously used for biological investigations, they were collected (day 0, days 1 and 2 of hospitalization, when available) from patients hospitalized for acute malaria symptoms occurring from mid September to end of December. Because of the limited capacity of the intensive care facility, we considered data in all patients consecutively admitted during 2 transmission seasons in 2000/2001 and 2002/2003. PCT determination was implemented for all the patients in 2000 2001 , but only for a subgroup of 33 randomly selected patients in 2002-2003 (for cost rea- 
sons of PCT assay). Recruitment and follow-up of severe malaria as well as individuals from endemic areas following the strict ad hoc ethic constraints has been previously reported..$^{8,9}$

The protocol was approved by the ad hoc Ethics Committee and informed consent for the use of previous withdrawal was obtained from patients or their parents explained in both French and in Wolof, the local language.

All patients selected for this study had confirmed cerebral malaria symptoms with impaired cerebral function (Glasgow score $<9$ ) when entering the intensive care facilities. ${ }^{4}$ Patients entering with fever and clinical signs of cerebral impairment underwent a lumbar puncture (to rule out meningitis) plus a thick blood smear, coma without detectable parasitaemia were confirmed for infection by use of immunochromatography test (ICT) detecting $P$. falciparum HRP2 (MaKromed, Johannesburg, South Africa). Some patients showed additional clinical or biological failures (metabolic acidosis, shock, pulmonary oedema, and renal failure). Patients with any type of serious infection (local abscess, microbial pharyngitis ...) before onset of cerebral malaria episode were excluded from the study, as summarised on Figure 1. None showed any positive blood culture during hospitalization.

The sequence of recruitment comprised 67 patients, including 16 fatal cases during the first transmission season, and 31 patients with 10 fatal cases during the second transmission season, leading to $26.5 \%$ of fatality rates. Levels of parasitaemia on day 0 were variable, and more than $50 \%$ of the patients had indeed received an antimalarial treatment before the onset of acute cerebral symptoms. Further description and characteristic of the patients are summarised in Table 1.

Anonymized blood samples were collected; plasma and red blood cells were separated by centrifugation, and stored at $-80^{\circ} \mathrm{C}$ until use.

\section{Measurement of procalcitonin}

The concentration of PCT in human serum by a commercial double site sandwich immunoluminometric assay according to manufacturer's intructions (LUMI-test ${ }^{\circledR}$ PCT; Brahms Diagnostica Gmbh, Berlin, Germany). Ab used recognized two distinct binding sites: the calcitonin and katacalcin segments. Plates pre-coated with one Ab were incubated with the sera and the other luminescent tracerlabelled $\mathrm{Ab}$. The excess of luminescent tracer was eliminated by washing before measuring the signal in a luminometer. The intensity of the luminescence signal (RLU) is related to the PCT concentration in the sample by using a calibration curve established using known standards for each set of measures.

\section{Statistical analysis}

Comparisons of PCT levels between different groups were done by the Mann-Withney rank test, the Wilcoxon signed rank test and the Spearman rank correlation test for nonnormally distributed data. P values $<0.05$ were considered significant. The ROC (Receiver Operating Characteristic) curves were calculated to determine the best cut-off point for PCT on admission and during the hospitalisa-

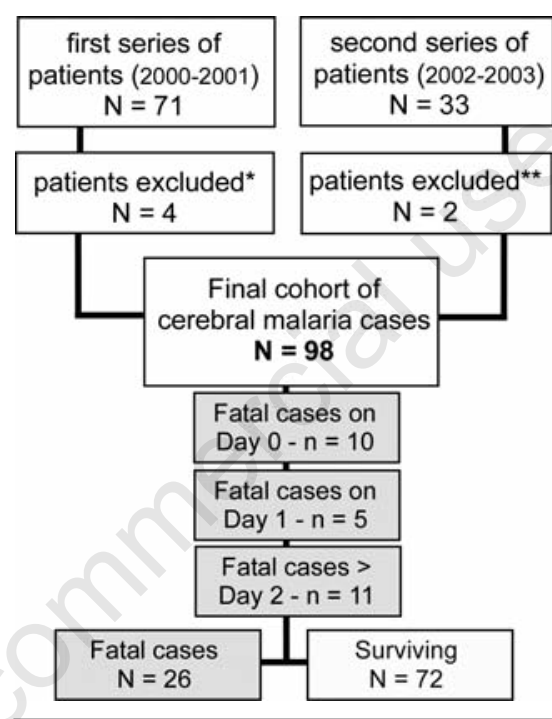

Figure 1. Summary of the recruitment for cerebral malaria patients. In this figure is summarized the sequence of recruitment of patients. 6 cases were excluded after examination of patient's files indicating a past of infection, which was also reflected by elevated level of PCT (mean level=196 nanog per $\mathrm{mL}$ ). Patients excluded from the first recruitment comprised 1 fatal outcome and showed a recent history of local abscess, microbial pharyngitis and pneumonia. Patients excluded from second recruitment were 2 fatal cases with a history of pulmonary infection by acinetobacter and pneumonia, respectively. tion and to compare AUC for PCT and haemoglobin. Logistic regression models were used to analyse the relationship between the survival of the patients and independent variables. The parameters of this statistical test were calculated. Statistical analyses were performed using Statview 5.0 ${ }^{\circledR}$ software (SAS Institute, Cary, NJ) and Egret 3.01 (Cytel).

\section{Results}

\section{Levels of PCT and cut-off values on day 0}

As shown on Figure 2A, distribution of PCT values measured on days 0,1 and 2 showed a large range of values from 0.1 to 280 nanog per $\mathrm{mL}$, with a general decrease on day 1 and 2 . As shown on Figure 2B, the distinction between surviving vs fatal cases showed a significant higher level of PCT on day 0 in fatal cases $(\mathrm{P}=0.0073)$, despite some individual low val-

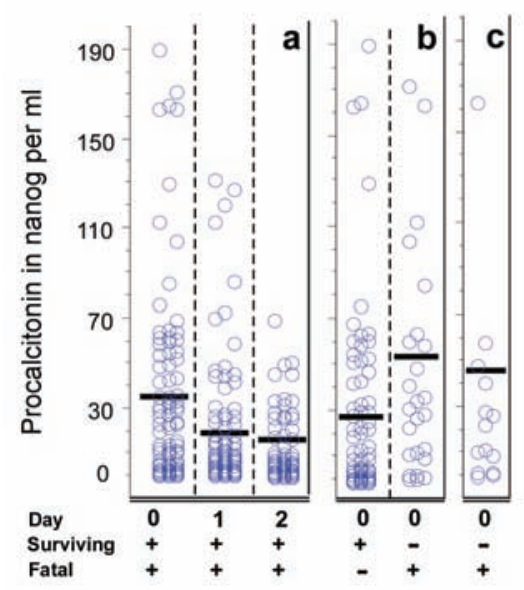

Figure 2. comparison of the distribution of PCT values in individuals from the 2 groups of patients. Scatter plot of the distribution of procalcitonin levels in individuals with cerebral malaria a) on day 0 of hospitalisation and days 1 and 2 after entrance in intensive care service; b) on day 0 for surviving vs fatal cases; $\mathrm{cc}$ ) patients deceased before day 2. Mean levels are plotted as thick lines.

Table 1. Description and characteristics of the patients with confirmed cerebral malaria.

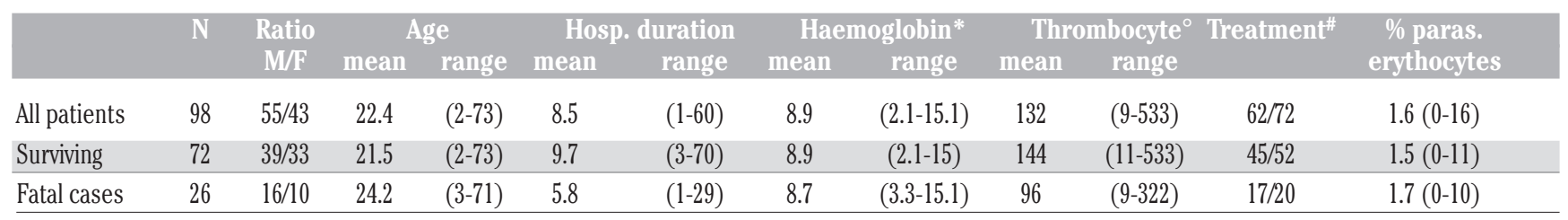

${ }^{*}$ Haemoglobin in $\mathrm{g} / 10^{-1} \mathrm{~L}$; ${ }^{\circ}$ Thrombocytes nx102/microlitre; ${ }^{\sharp}$ numbers of individuals that declared or received an anti-malarial treatment before oneset of cerebral manifestations. 
ues. Of note, individuals with fatality on day 0 did not have significant higher levels of PCT than the overall set of fatal cases (Figure 2C).

Using the cut off of $69 \mathrm{ng} / \mathrm{mL}$ on day 0 , PCT measure had a specificity of $93.06 \%$ (95\% CI= 84.5-97.7) for predicting mortality; however, it had a low sensitivity (sensitivity 23.08\%, 95\% CI: 9.0-43.7). In addition, the interest of PCT measure was assessed by comparison with haemoglobin concentration, a known marker of severity (Figure 3B). Mortality could be most accurately predicted by $\mathrm{PCT}$ at day 0 $(\mathrm{AUC}=0.694)$ then by haemoglobin (AUC = 0.511: z statistic: $\mathrm{P}=0.046)$. This result was confirmed using logistic regression models. The following independent variables were included in the full model (PCT at day 0, haemoglobin, platelets, age and gender). Using backward selection procedure, the final model included only PCT at day0 [Coefficient $=0.012, \mathrm{P}=0.019 ; \mathrm{OR}=1.0123$ (CI95\%, 1.0021.023) for $1 \mathrm{ng} / \mathrm{mL}$ of PCT]. There was no evidence of significant correlation between PCT levels with: i) parasitaemia measured on day 0 ; ii) age of individuals. Interestingly, age positively correlated with parasitaemia (rho $=0.33$, $\mathrm{P}<0.01$ ), underlining a potential higher parasite load in older individuals, possibly related with a more systematic treatment of febrile infants by their parents or relatives before reaching the hospital.

\section{Comparison and kinetics of PCT levels between surviving and fatal cases}

As shown in Table 2, a marked significant lower levels of PCT was found in non-fatal cerebral malaria cases for the overall patients during the 3 days of post hospitalisation followup $(\mathrm{P} \leq 0.01)$. Mean and median values were $50 \%$ and $70 \%$ lower in surviving individuals than fatal cases, respectively.

Shown on Figure 3A is the kinetics of PCT measures in surviving and fatal cases. There was a clear tendency toward decreasing levels of PCT from day 0 to day 2 in surviving patients, where levels of PCT are found significantly lower day after day $(\mathrm{P}<0.001)$, with a mean daily decrease over $40 \%$. Importantly, in all surviving individuals with initial PCT values $>69.98 \mathrm{ng}$ per $\mathrm{mL}(\mathrm{n}=5 / 72)$, a substantial decrease was evidenced within day 2 .

According to ROC curves (Figure 3B), the best cut point for PCT on the overall set of values on days 0,1 and 2 was $>27 \mathrm{ng}$ per $\mathrm{mL}$. This cut point showed an acceptable combination of specificity [63.89 (95\% CI=51.7-74.9)], with a reasonable sensitivity $[69.23 \%(95 \% \mathrm{CI}=48.2$ $85.6)$ ]. Using this cut off, only 8 of the 26 deaths (30.8\%) would not have been predicted.

\section{Discussion}

This study aimed to investigate PCT circulating levels in individuals living in an urban area of low endemicity, who developed cerebral malaria following $P$. falciparum infection. Two study-groups of patients, to be considered as very partially immune but not naive, were enrolled in a retrospective recruitment, and categorized on the basis of the clinical outcomes. These individuals, from the same location during different time periods regardless of previous exposure to infective bites and variable history of malaria episode, shared similar observable clinical outcomes of cerebral malaria resulting from a similar risk for clinical episodes. Recruitment was limited to the transmission season and restricted by the capacity of the intensive care facilities, requiring a cumulative sample collection during consecutive seasons for an adequate number of patients. Indeed, a set of non-parasitic factors contributing to fatality cannot be excluded; nevertheless comparable situation exists also in the non-fatal cases sustaining the comparison between the two groups of patients: fatal vs surviving. In addition, whatever the initial clinical failure due to malaria episode when entering hospital, all patients were equally treated in the most adequate facility in Dakar.

This study confirmed the observation that high levels of PCT is associated with P.falciparum active infection; previous studies reported mean PCT level $>10-20 \mathrm{ng} / \mathrm{mL}$ in patients of all age suffering of malaria symptoms. ${ }^{10-13}$ In addition, this tendency for high levels of PCT on admission at hospital results from substantially large range of values. On the other side, recent appraisal of procalcitonin as a biomarker for severe $P$. falciparum disease showed high negative predictive value of both neopterin and procalcitonin for a rapid exclusion of severe malaria disease on admission in hospitals from northern country. ${ }^{14}$

Importantly, results found in this study were in close agreement with those published in Gabonese children, ${ }^{12}$ with PCT levels of 25.2 $\mathrm{ng} / \mathrm{mL}$ in acute malaria vs $77 \mathrm{ng} / \mathrm{mL}$ in cerebral malaria, and were slightly higher than those recently reported in severe malaria cases (18.8 \pm 11.6 ) in 18 Ghanaian children. ${ }^{15}$

The present investigation assessed that PCT is a prognostic marker of complicated $P$. falciparum infection. Indeed pathologic process relates with the parasite load of the patient,

Table 2. Comparison of procalcitonin levels between patients with fatal and non-fatal cerebral malaria.

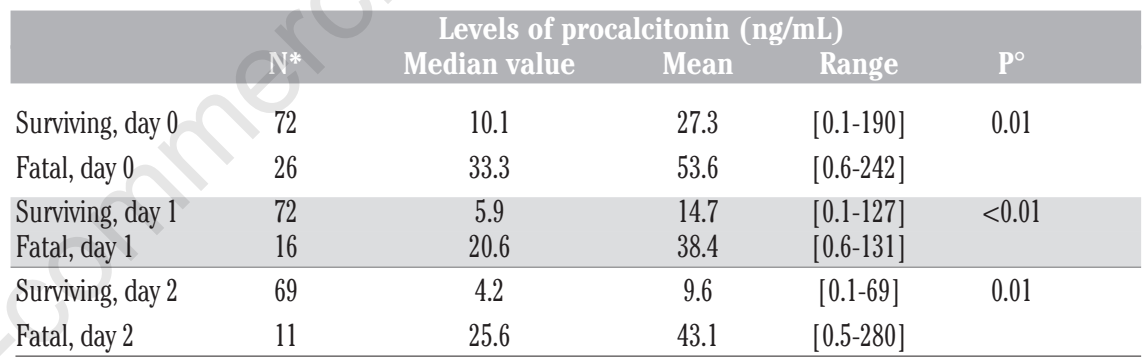

*Number of determinations on withdrawals available on days 0,1 and $2 ;{ }^{\circ}$ comparison of Procalcitonin levels between surviving $v$ fatal cases.
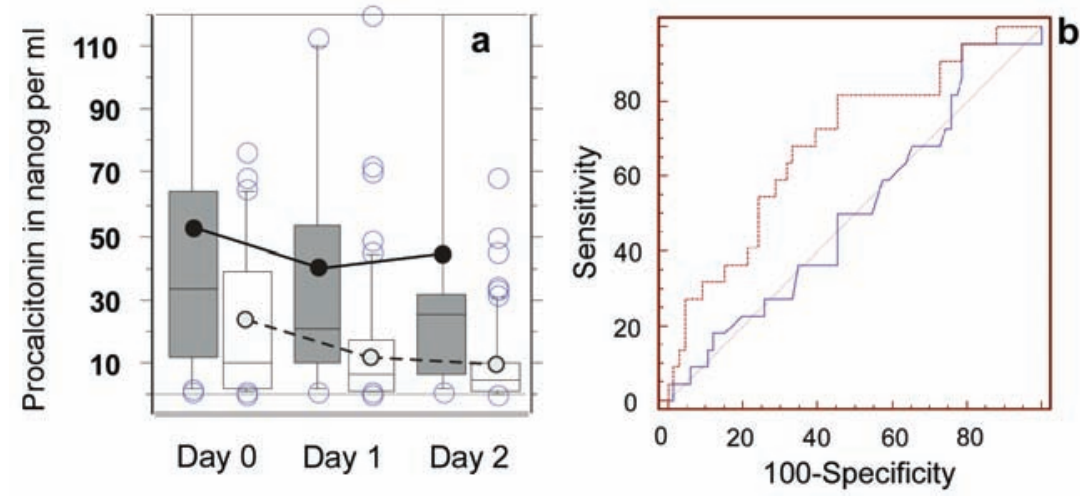

Figure 3. Kinetic and ROC curves of PCT values in individuals with cerebral malaria. Shown are the magnitude and kinetic of individual level of PCT on day 0,1 and 2 as box plot (a). Fatal cases (dark grey) show higher levels and less decrease of PCT values than surviving individuals (light grey). Mean values are plotted as plain lines (fatal cases) and dashed lines (surviving). In part b) is shown the ROC curves for PCT values (dashed) and haemoglobin (plain) plotting sensitivity as function of 100 -specificity. Optimal values are $69.2 \mathrm{ng} / \mathrm{mL}$ for specificity, and $30 \mathrm{ng} / \mathrm{mL}$ for both specificity and sensitivity. 
reflected by the circulating parasitaemia. A significant relationship between PCT levels, parasitaemia and the degree of severity was observed in some studies, especially in nonimmune patients recruited in hospitals from northern country. ${ }^{4}$ This was not observed in this study, based on a recruitment of individuals living in endemic country: they usually receive antimalarial treatment early in case of fever, a situation that could lead to manifestations of cerebral malaria without detectable parasitaemia by blood smear when entering the intensive care facility.

Multivariate analysis (including haemoglobin, platelet counts, age and gender) underlined PCT concentration at day 0 as a better predictive parameter for fatality in this study. Indeed, recent studies showed that platelets contribute to immune activation as part of the pathogenesis of cerebral malaria in experimental models. ${ }^{16}$ In addition, low platelet counts have been found associated with CM, and the degree of thrombocytopenia was observed directly proportional to the density of parasitaemia. ${ }^{17}$ We did not underline relationship between platelet counts and severity in this study, high PCT levels may possibly reflect such inflammation-related effect. Further analyses are required for hospitalized patients to clarify relationship between thrombocytopenia and fatality in CM.

Studies by others examined the initial picture of inflammation and the levels of inflammation-related markers such as cytokines. Their studies showed some relevance with severity but were not clearly established as a prognostic value in fatal cases: high levels of IL6 and tumor necrosis factor have been reported in cerebral malaria with substantial decrease measured on day 3 to $7 .^{12,18,19}$ There were rather some significant differences in severe anaemia cases, where IL6 and IL10 levels were found lower than other groups of severe malaria cases. ${ }^{18,20}$ A recent work, having reported on a large set of 36 different biomarkers in cerebrospinal fluids from fatal cerebral malaria cases, ${ }^{21}$ concluded in the inability to delineate a clear marker of fatality. Another recent work examining 11 host biomarkers in Ugandan children underlined a combination of two soluble factors from myeloid cells and endothelial activation as a relevant predictive marker of fatal outcom. ${ }^{22}$ However, this study requires further confirmation and may not be applicable to adults as studied here. These previous data have underlined the complex relationships between inflammatory events, cell activation, cytokines release and disease.

In our present study, the individual level of PCT does not predict a fatal issue, despite high initial levels and a cut-off point close to $70 \mathrm{ng}$ per mL. The picture of a significant initial threshold of PCT before fatal outcome was evi- denced in naive hospitalized patients where 6 out of 7 cases had twice-higher levels of PCT than recovering patients. Interestingly, the mean level reported was also close to $70 \mathrm{ng}$ per mL. Of note, recovering patients that had consistently PCT levels increasing on day +1 , decreased on day +2 , possibly in relation with the variation of parasite load. ${ }^{4}$ However, the absence of decrease within the second day of observation period echoes as an alarm for the clinician, with serious possibility of fatality by day 3 and onwards (data not shown).

\section{Conclusions}

In conclusion, Procalcitonin (PCT), a clinical marker for sepsis and systemic inflammatory reactions, has a clear value in order to follow-up severe malaria in semi-immune patients, and predict fatal evolution ever in immune individuals with a complex past of infection and unknown treatment before hospitalisation. In these cases, values of the two cut-off points 70 and $30 \mathrm{ng}$ per ml (on day 0 and during the 3 days of observation, respectively), together with a longitudinal follow-up are of strong interest for predicting mortality. Above these values, a lack of early decrease in PCT levels may not witness a future recover of the patient. Without observable decrease, clinicians will closely survey the monitoring of the patient, accompanied by an adaptation of the treatment (modifications of doses and/or combination with other antimalarial or antibiotics) and anticipate and discuss with the medical team for possible additive interventions such as renal dialysis or blood transfusion. This study indicated that PCT is useful in monitoring cerebral malaria patients and in predicting mortality.

\section{References}

1. Russwurm S, Wiederhold M, Oberhoffer M, et al. Molecular aspects and natural source of procalcitonin. Clin Chem Lab Med 1999,37:789-97.

2. Assicot M, Gendrel D, Carsin H, et al. High serum procalcitonin concentrations in patients with sepsis and infection. Lancet 1993;341:515-8.

3. Dandona P, Nix D, Wilson MF, et al. Procalcitonin increase after endotoxin injection in normal subjects. J Clin Endocrinol Metab 1994;79:1605-8.

4. Chiwakata CB, Manegold C, Bonicke L, et al. Procalcitonin as a parameter of disease severity and risk of mortality in patients with Plasmodium falciparum malaria. J Infect Dis 2001;183:1161-4.
5. Pages F, Texier G, Pradines B, et al. Malaria transmission in Dakar: a two-year survey. Malar J 2008;7:178.

6. Trape JF, Lefebvre-Zante E, Legros F, et al. Vector density gradients and the epidemiology of urban malaria in Dakar, Senegal. Am J Trop Med Hyg 1992;47:181-9.

7. Diallo S, Konate L, Ndir O, et al. Malaria in the central health district of Dakar (Senegal). Entomological, parasitological and clinical data. Sante 2000;10:221-9.

8. Perraut R. Evaluation of anti-Plasmodium falciparum antibodies in Senegalese adults using different types of crude extracts and recombinant antigens. Microbes Infect 2002;4:31-5.

9. Perraut R, Diatta B, Marrama L, et al. Differential antibody responses to Plasmodium falciparum glycosylphosphatidylinositol anchors in patients with cerebral and mild malaria. Microbes Infect 2005;7:682-7.

10. Davis TM, Assicot M, Bohuon C, et al. Serum procalcitonin concentrations in acute malaria. Trans R Soc Trop Med Hyg 1994;88:670-1.

11. Hollenstein U, Looareesuwan S, Aichelburg A, et al. Serum procalcitonin levels in severe Plasmodium falciparum malaria. Am J Trop Med Hyg 1998;59:860-3.

12. Richard-Lenoble D, Duong TH, Ferrer A, et al. Changes in procalcitonin and interleukin 6 levels among treated African patients with different clinical forms of malaria. Trans R Soc Trop Med Hyg 1997;91:305-6.

13. Hesselink DA, Burgerhart JS, BosmansTimmerarends $\mathrm{H}$, et al. Procalcitonin as a biomarker for severe Plasmodium falciparum disease: a critical appraisal of a semi-quantitative point-of-care test in a cohort of travellers with imported malaria. Malar J 2009;8:206.

14. te Witt R, van Wolfswinkel ME, Petit PL, et al. Neopterin and procalcitonin are suitable biomarkers for exclusion of severe Plasmodium falciparum disease at the initial clinical assessment of travellers with imported malaria. Malar J 2010;9:255.

15. Braun N, Marfo Y, Von Gartner C, et al. CTLA-4 positive T cells in contrast to procalcitonin plasma levels discriminate between severe and uncomplicated Plasmodium falciparum malaria in Ghanaian children. Trop Med Int Health 2003;8:1018-24.

16. Srivastava K, Cockburn IA, Swaim A, et al. Platelet factor 4 mediates inflammation in experimental cerebral malaria. Cell Host Microbe 2008;4:179-87.

17. Wassmer SC, Taylor T, Maclennan CA, et al. Platelet-induced clumping of Plasmodium falciparum-infected erythrocytes from Malawian patients with cere- 
bral malaria-possible modulation in vivo by thrombocytopenia. J Infect Dis 2008; 197:72-8.

18. Lyke KE, Burges R, Cissoko Y, et al. Serum levels of the proinflammatory cytokines interleukin-1 beta (IL-1beta), IL-6, IL-8, IL10, tumor necrosis factor alpha, and IL12(p70) in Malian children with severe Plasmodium falciparum malaria and matched uncomplicated malaria or healthy controls. Infect Immun 2004;72:5630-7.
19. Sarthou JL, Angel G, Aribot G, et al. Prognostic value of anti-Plasmodium falciparum-specific immunoglobulin G3, cytokines, and their soluble receptors in West African patients with severe malaria. Infect Immun 1997;65:3271-6.

20. Kurtzhals JA, Adabayeri V, Goka BQ, et al. Low plasma concentrations of interleukin 10 in severe malarial anaemia compared with cerebral and uncomplicated malaria. Lancet 1998;351:1768-72.
21. Armah HB, Wilson NO, Sarfo BY, et al. Cerebrospinal fluid and serum biomarkers of cerebral malaria mortality in Ghanaian children. Malar J 2007;6:147.

22. Erdman LK, Dhabangi A, Musoke C, et al. Combinations of host biomarkers predict mortality among Ugandan children with severe malaria: a retrospective case-control study. PLoS One 2011;6:e17440. 